\title{
SIGNIFICADO CLÍNICO DA HIPERTENSÃO DURANTE O SONO
}

\section{CLINICAL MEANING OF HYPERTENSION DURING SLEEP}

Fernando Nobre1, Décio Mion Júnior ${ }^{2}$

\section{RESUMO}

A anormalidade da pressão arterial durante o período de sono identificada como médias $\geq 120 \times 70 \mathrm{~mm} H \mathrm{Hg}$, por meio de registros da Monitorização Ambulatorial da Pressão Arterial de 24 horas (MAPA), está relacionada a pior prognóstico e maior risco de eventos. Essa alteração pode ser decorrência de vários fatores, mas, geralmente, independentemente da causa está fortemente relacionada a maior probabilidade de eventos e mortalidade cardiovasculares. Ainda restam dúvidas, embora evidências começam a ser oferecidas, se o tratamento medicamentoso desse estado de comportamento peculiar da pressão arterial nas 24 horas deva ser instituído. Nessa revisão esses aspectos são amplamente discutidos com base nas melhores evidências disponíveis.

Descritores: Monitorização Ambulatorial da Pressão Arterial (MAPA); Pressão Arterial; Hipertensão; Sono.

\section{ABSTRACT}

The abnormality of blood pressure during the sleep period identified as means $\geq 120 \times 70 \mathrm{~mm} \mathrm{Hg}$, through 24-hour Ambulatory Blood Pressure Monitoring (ABPM) records, is related to a worse prognosis and greater risk of events. This change can be due to several factors, but, generally, regardless of the cause, it is strongly related to a higher probability of cardiovascular events and mortality. Doubts remain, although evidence is beginning to be offered, whether drug treatment of this peculiar behavioral state of blood pressure within 24 hours should be instituted. In this review, these aspects are widely discussed based on the best available evidence.

Keywords: Ambulatory Blood Pressure Monitoring (ABPM); Arterial Pressure; Hypertension; Sleep.

A Monitorização Ambulatorial da Pressão Arterial (MAPA) introduzida como método de avaliação da Pressão Arterial (PA) fora da clínica, na década de 1960, ganhou destaque nos últimos anos por várias razões, mas principalmente por ser o único método que possibilita medidas da PA durante o sono.

Em 2003, O’Brien et al. ${ }^{1}$ definiram vários padrões de comportamentos da PA que puderam ser definidos com base nos registros obtidos da pressão arterial obtidos pela MAPA.

Foram considerados, então, sete comportamentos da PA, dentre eles a Hipertensão Arterial Noturna representada por comportamento anormal durante o período de sono.

A elevação da PA durante o sono pode ser fruto da falta de descenso durante esse período quando se avalia o ritmo circadiano da PA pela MAPA, sendo esse fenômeno mediado por vários mecanismos.

\section{CONCEITO}

Apesar de haver discordâncias entre as diretrizes europeias e americanas quanto ao valor de anormalidade da pressão arterial durante o sono, avaliada pela MAPA, consideraremos os critérios definidos pela Diretriz Europeia de MAPA ${ }^{2,3}$ que estabelecem como anormais valores maiores ou iguais a $120 \times 70$ mm Hg no sono com valores normais de PA na vigília $(<135 \times 85 \mathrm{~mm} \mathrm{Hg})$. (Figura 1)

\section{EPIDEMIOLOGIA}

A prevalência da hipertensão arterial noturna varia entre as diferentes populações em decorrência de características demográficas, clinicas e étnicas, além dos critérios de anormalidade adotados na avaliação. ${ }^{4}$

Análises retrospectivas mostraram prevalência de hipertensão durante o sono alta em sul-africanos (10,2\%) e em japoneses $(10,9 \%)$ com variações entre $6 \%$ e $7,9 \%$ entre europeus. ${ }^{5}$

\section{SIGNIFICADO CLÍNICO E PROGNÓSTICO}

Sabe-se que, do ponto de vista de prognóstico, o ideal é que haja uma redução da PA de 10 a 20\% durante o sono quando comparada à vigília.

1. Unidade de Hipertensão do Hospital das Clínicas da Faculdade de Medicina da USP de Ribeirão Preto, SP, Brasil.

2. Hospital das Clínicas da Faculdade de Medicina da USP (HCFMUSP), São Paulo, SP, Brasil.

Correspondência: Av. Independência, 3767, CEP 14O26-150, Ribeirão Preto, SP, Brasil. fernando.nobre@uol.com.br

http://dx.doi.org/10.4787O/1519-7522/2O2O2704130-3 
Quando são avaliadas pressões arteriais obtidas por meio de diversas formas constata-se que os valores resultantes das medidas durante o sono são os que melhor se correlacionam com mortalidade. (Figura 2$)^{6}$

A falta de redução da pressão arterial enquanto se dorme em relação ao período de vigília e a sua elevação estão relacionadas a pior prognóstico. ${ }^{7}$ (Figura 3)

Vários estudos demonstraram essa correlação entre PA alterada durante o sono e prognóstico, como expresso na Tabela 1.

Entre as várias possibilidades de comportamento em relação às variações entre vigília e sono podemos ter as seguintes possibilidades: descenso normal (entre 10 e $20 \%$ ), atenuado ( $>0 \%$ e $<10 \%$ ), ausente $(\leq 0 \%)$ e acentuado ( $>20 \%$ ), segundo as Diretrizes Brasileiras de MAPA. ${ }^{3}$ (Tabela 2 )

Tabela 2. Diversos tipos de comportamento entre as pressões na vigília e no sono. ${ }^{3}$

Prognósticos de acordo com esses diversos comportamentos podem ser observados na Figura 4.

Pode-se observar que tanto a falta de descenso quanto a elevação da pressão arterial quando comparados os períodos de vigília e sono se correlacionam a pior prognóstico estimado por avaliação da mortalidade total ao longo do tempo como a ocorrência de eventos cardiovasculares.

Esse padrão na realidade expressa o oposto que se espera quando é avaliado o ritmo nictemérico da pressão arterial.

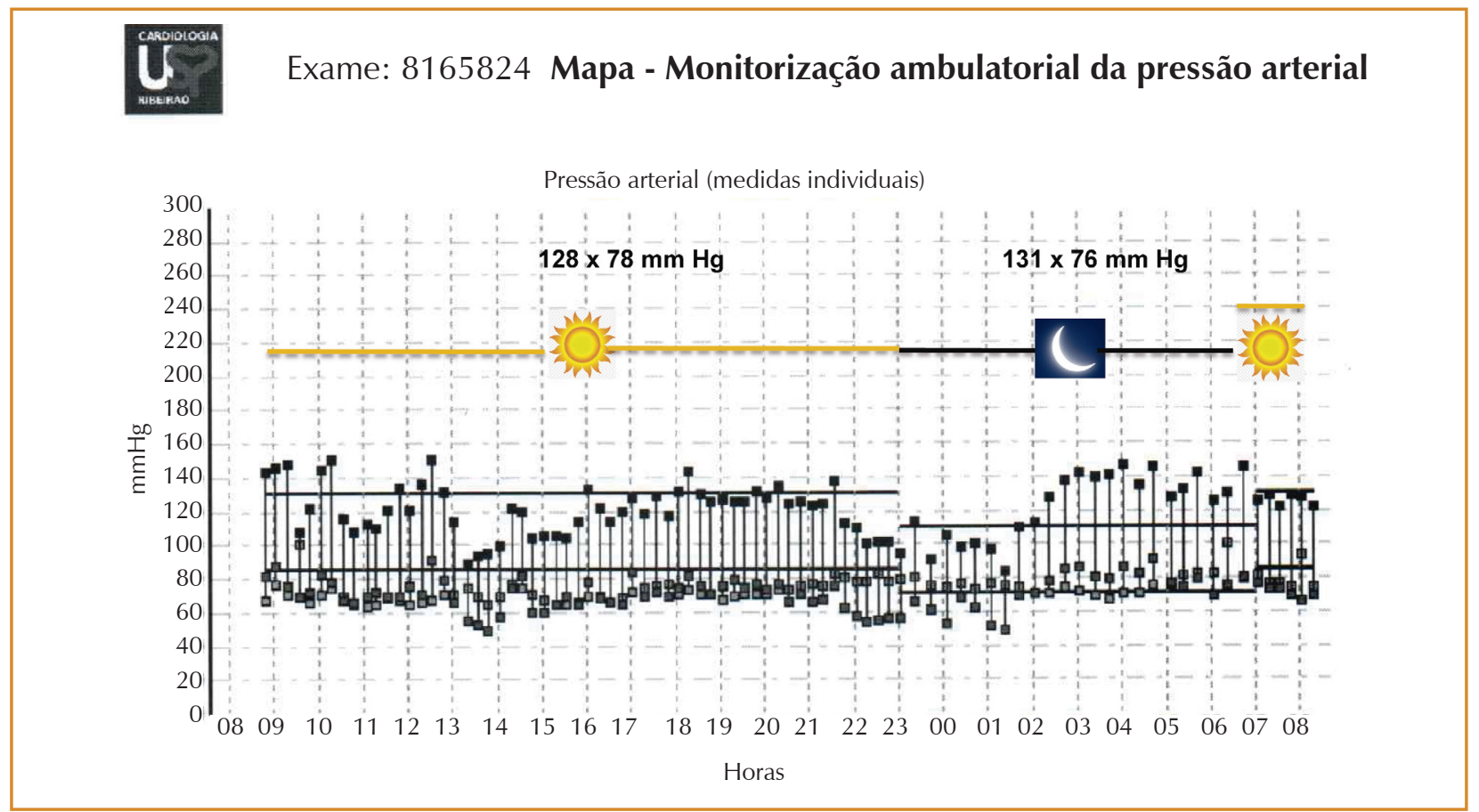

Figura 1. MAPA de 24 horas com comportamento anormal da pressão arterial durante o período de sono com médias de $131 \times 76 \mathrm{~mm} \mathrm{Hg}$. (considerando-se anormais médias nesse período $\geq 120$ x $70 \mathrm{~mm} \mathrm{Hg}$ ).
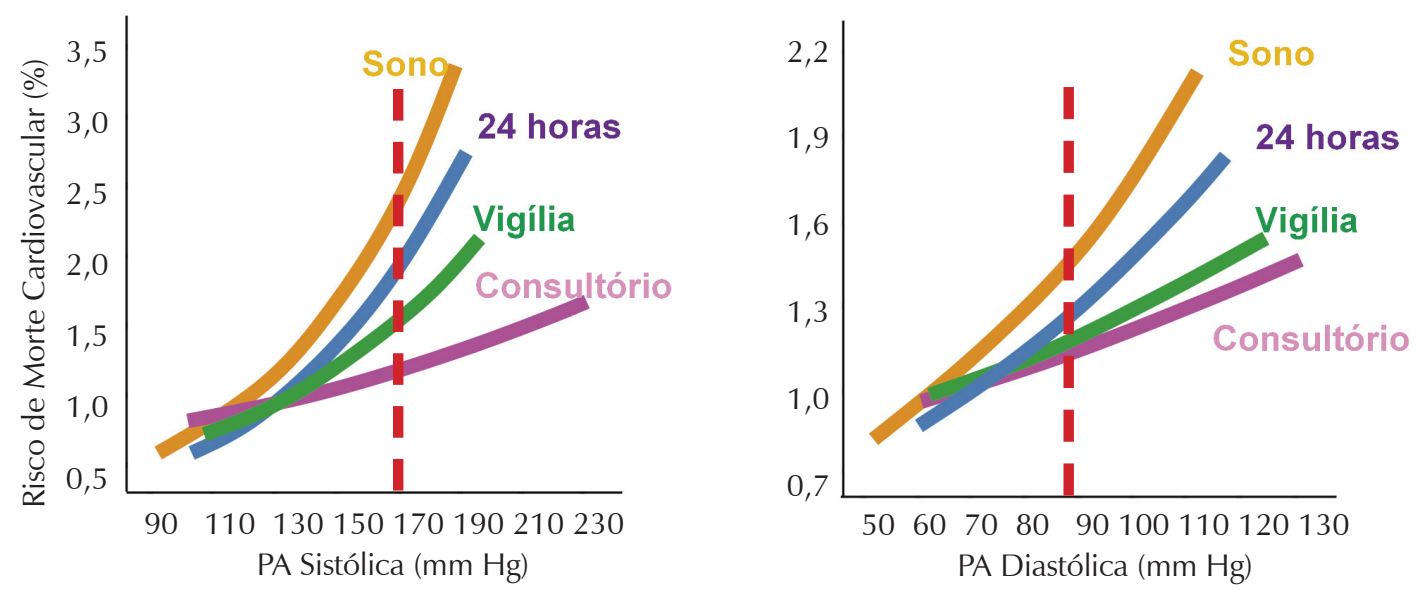

Figura 2. Mortalidade cardiovascular de acordo com as Pressões Arteriais Sistólica e Diastólica medidas em consultório e pela MAPA (vigília, 24 horas e sono). ${ }^{6}$ 


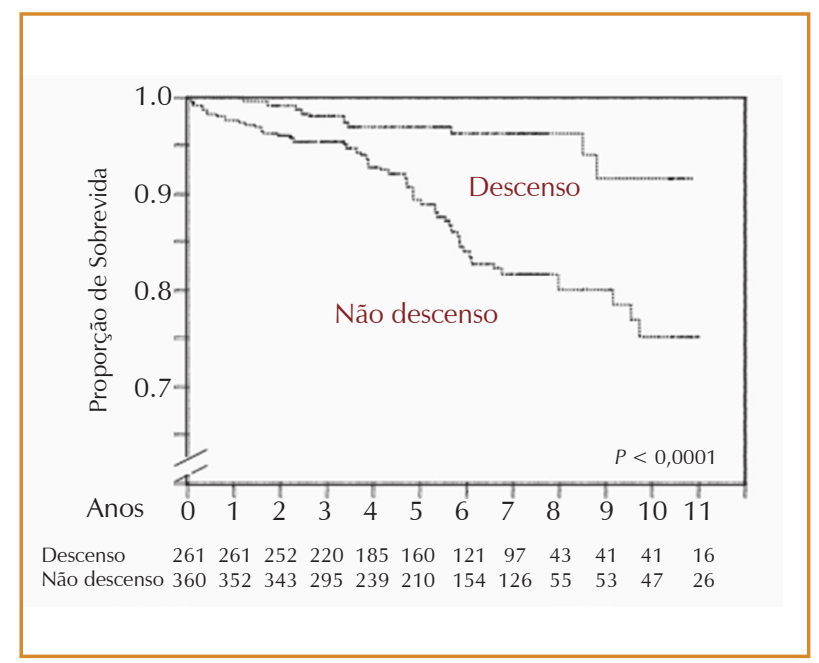

Figura 3. Prognóstico estimado pela sobrevida durante anos de seguimento de acordo com a ocorrência de descenso ou não. ${ }^{8}$

Tabela 1. Prognóstico da Hipertensão Arterial Noturna segundo alguns estudos.

\begin{tabular}{l|c|l}
\multicolumn{1}{c|}{ Estudos } & n & \multicolumn{1}{c}{ Desfechos } \\
$\begin{array}{l}\text { Hypertens. 2010;28(10): } \\
\text { 2036-2045 }\end{array}$ & 8.000 & $\begin{array}{l}\text { Alto risco de Eventos } \\
\text { Cardiovasculares e } \\
\text { Mortalidade Total }\end{array}$ \\
\hline J Am Heart Assoc. & - & $\begin{array}{l}\text { Eventos decorrentes } \\
\text { de DRC e } \\
\text { Cardiovasculares }\end{array}$ \\
\hline $\begin{array}{l}\text { J Clin Hypertens } \\
\text { (Greenwich). } 2018 ; 20\end{array}$ & - & $\begin{array}{l}\text { Descenso Reverso } \\
\text { relacionado com } \\
\text { (9):1238-1246 }\end{array}$ \\
\hline
\end{tabular}

DRC = Doença Renal Crônica; AVE = Acidente Vascular Encefálico.

Tabela 2. Diversos tipos de comportamento entre as pressões na vigília e no sono. ${ }^{3}$

\begin{tabular}{l|c}
\multicolumn{1}{c|}{ Descenso presente } & $\geq \mathbf{1 0} \leq \mathbf{2 0}$ \\
\hline Descenso ausente ou ascensão da PA & $\leq 0$ \\
\hline Atenuado & $>0$ e $<10$ \\
\hline Descenso acentuado & $>20 \%$ \\
\hline
\end{tabular}

\section{CONDUTAS}

A despeito do claro risco demonstrado particularmente com a ausência de descenso dentro dos padrões desejados com variações entre 10 a $20 \%$ de queda da pressão arterial entre a vigília e o sono e da presença de hipertensão arterial nesse período ainda não está absolutamente definido se intervenções medicamentosas que determinem a redução da pressão arterial, são benéficas e devam ser instituídas.

O estudo MAPEC ${ }^{11}$ comparou a administração de medicamentos pela manhã e ao deitar-se, após um seguimento de 5,6 anos em 2156 pacientes com hipertensão observou-se que a usar medicamento antes de dormir resultou em melhor controle da pressão arterial e menor risco de doenças cardiovasculares comparados com os que tomavam as medicações apenas pela manhã.
A

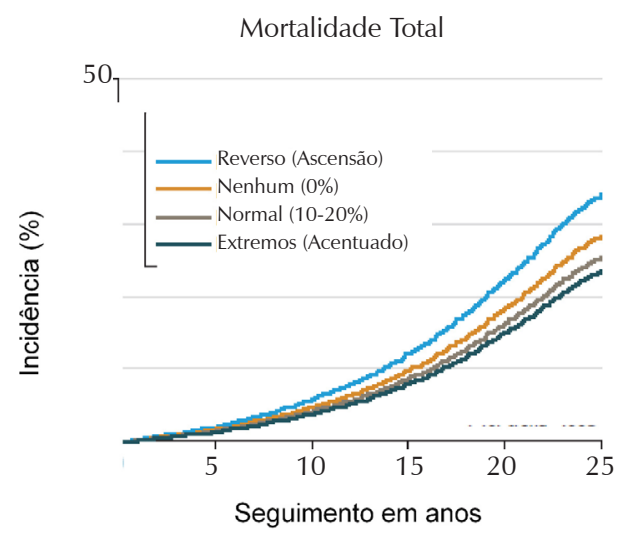

B

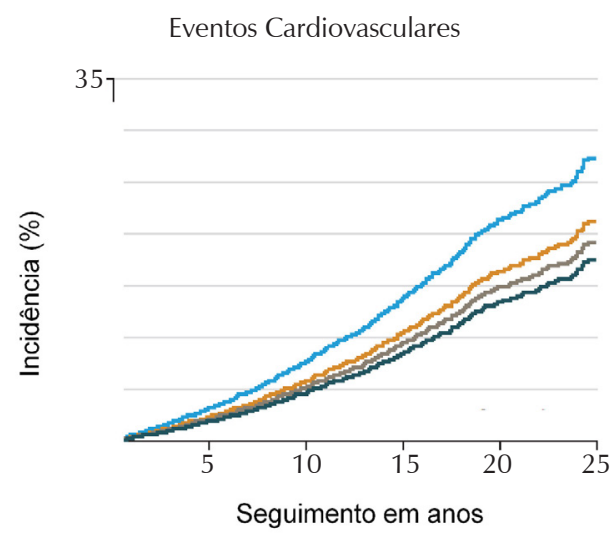

Figura 4. Prognóstico de acordo com os diferentes tipos de comportamento da pressão arterial entre os períodos de vigília e sono. ${ }^{9}$

Ainda foi observado que a prevalência de falta de descenso foi reduzida de $62 \%$ para $34 \%$.

Alguns autores mostraram que a restrição de sal e emprego de diuréticos reduz significativamente a pressão arterial durante o sono chegando mesmo a determinar que pacientes sem descenso passassem a apresentá-lo. ${ }^{12,13}$

Devido aos mecanismos de ação e aspectos fisiopatológicos possivelmente envolvidos na ocorrência da hipertensão arterial durante o sono é possível que IECAs e BRAs associados com diuréticos possam ter benefícios superiores ao emprego de outros medicamentos anti-hipertensivos. ${ }^{4}$

Apesar de não se ter ainda a definição de condutas com base em estudos de intervenções e conclusões decorrentes de observações clínicas bem conduzidas, tudo leva a crer que o controle das elevações da pressão arterial durante o sono e a sua correção deva ser adotado. 


\section{REFERÊNCIAS}

1. O'Brien E, Asmar R, Beilin L, et al; European Society of Hypertension Working Group on Blood Pressure Monitoring. European Society of Hypertension recommendations for conventional, ambulatory and home blood pressure measurement. J Hypertens. 2003;21(5):821-48. doi: 10.1097/00004872-200305000-00001.

2. Williams B, Mancia G, Spiering W, et al.; ESC Scientific Document Group. 2018 ESC/ESH Guidelines for the management of arterial hypertension. Eur Heart J. 2018;39:3021-104. doi: 10.1093/eurheartj/ehy339.

3. Nobre F, Mion Jr. D, Gomes MAM, et al. 6- Diretrizes de Monitorização Ambulatorial da Pressão Arterial e 4⿳亠口冋 Diretrizes de Monitorização Residencial da Pressão Arterial. Arq BrasCardiol. 2018;110(5Supl.1):1-29. doi: 10.5935/abc.20180074

4. Tadic M Cuspidi C, Grassi G, Mancia G.. Isolated Nocturnal Hypertension: What Do We Know and What Can We Do? Integr Blood Press Control.2020;13:63-9. doi: 10.2147/IBPC.S223336

5. Li Y, Wang JG. Isolated nocturnal hypertension: a disease masked in the dark. Hypertension. 2013;61:278-83. doi: 10.1161/ HYPERTENSIONAHA.111.00217.

6. Dolan E, Stanton A, Thijs L, et al. Superiority of ambulatory over clinic blood pressure measurement inpredicting mortality: the Dublin outcome study. Hypertension. 2005 Jul;46(1):156-61. doi: 10.1161/01. HYP.0000170138.56903.7a.

7. Nobre F, Mion Junior D. Ambulatory Blood Pressure Monitoring: Five Decades of More Light and Less Shadows. Arq Bras Cardiol. 2016:106(6):528-37.doi: 10.5935/abc.20160065.
8. Brotman DJ, Davidson MB, Boumitri M, Vidt DG. Impaired diurnal blood pressure variation and all-cause mortality. Am J Hypertens. 2008 Jan;21(1):92-7. doi: 10.1038/ajh.2007.7.

9. Yang WY, Melgarejo JD, Thijs L, et al.; International Database on Ambulatory Blood Pressure in Relation to Cardiovascular Outcomes (IDACO) Investigators. Association of Office and Ambulatory Blood Pressure With Mortality and Cardiovascular Outcomes. JAMA. 2019;322(5):409-20. doi: 10.1001/jama.2019.9811.

10. Salles GF, Reboldi G, Fagard RH, et al. Prognostic Effect of the Nocturnal Blood Pressure Fall in Hypertensive Patients: The Ambulatory Blood Pressure Collaboration in Patients With Hypertension (ABC-H) MetaAnalysis. Hypertension. 2016 Apr;67(4):693-700. doi: 10.1161 HYPERTENSIONAHA.115.06981.

11. Hermida RC, Diana EA, Artemio M, et al. Influence of circadian time of hypertension treatment on cardiovascular risk: results of the MAPEC Study. Chronobiol Int. 2010;27(8):1629-51. doi: 10.3109/07420528.2010.510230.

12. Uzu T, Ishikawa K, Fujii T, Nakamura S, Inenaga T, Kimura G. Sodium restriction shifts circadian rhythm of blood pressure from nondipper to dipper in essential hypertension. Circulation. 1997;96 (6):1859-62. doi: 10.1161/01.cir.96.6.1859.

13. Uzu T, Kimura G. Diuretics shift circadian rhythm of blood pressure from nondipper to dipper in essential hypertension. Circulation. 1999;100(15):1635-8. doi: 10.1161/01.cir.100.15.1635 